\title{
THE CARTAN MATRIX OF A GROUP ALGEBRA MODULO ANY POWER OF ITS RADICAL
}

\author{
PETER LANDROCK ${ }^{1}$
}

\begin{abstract}
We prove that the Cartan matrix of a group algebra $F[G]$ modulo any power of its radical $J$ is dual symmetric, provided $F$ is a splitting field of $F[G] / J$. This eases the process of determining the Loewy series of the projective indecomposable $F[G]$-modules.
\end{abstract}

Let $G$ be any finite group and $F$ a field of characteristic $p$. By a module of the group algebra $F[G]$, we will always mean a right module. Let $S_{1}, S_{2}, \ldots, S_{k}$ be a complete set of representatives of the isomorphism classes of simple $F[G]$-modules, and let $P_{i}$ be the projective cover of $S_{i}$. For any $F[G]$-module $M$, we let $M^{*}$ denote the dual $F[G]$-module of $M$. The dual of $S_{i}$ is denoted by $S_{i^{*}}$ as well. Thus $P_{i}^{*}=P_{i^{*}}$, as $F[G]$ is symmetric. In fact we will only use this fundamental property of a group algebra in the following. Consequently, similar results hold for symmetric algebras rather than just group algebras.

In the following we set $A=F[G]$ and denote its radical by $J$. As usual, the Loewy length of an $A$-module $M$ is the minimal number $r$ for which $M J^{r}=0$. Observe that $M$ and $M^{*}$ always have the same Loewy length, as $M=M^{* *}$.

From now on we must assume $F$ is a splitting field.

Now let $I$ be any power of $J$ and set $\bar{A}=A / I, \bar{P}_{i}=P_{i} / P_{i} I$. As $S_{i} I=0$ for all $i$, $\left\{S_{1}, S_{2}, \ldots, S_{k}\right\}$ is also a complete set of representatives of isomorphism classes of simple $\bar{A}$-modules of the Artinian algebra $\bar{A}$. Recall that the Cartan matrix $\left\{c_{i j}^{-}\right\}$of $\bar{A}$ is defined as follows: $c_{i j}^{-}$equals the multiplicity of $S_{j}$ as a composition factor of $\bar{P}_{i}$, which conveniently equals $\operatorname{dim}\left(\operatorname{Hom}_{\bar{A}}\left(\bar{P}_{j}, \bar{P}_{i}\right)\right)$ (see [1]). It is also well known that the Cartan matrix of $A$ is symmetric. The aim of this note is to prove

Theorem A. The Cartan matrix $\left\{c_{i j}^{-}\right\}$of $\bar{A}$ is dual symmetric, i.e. $c_{i j}^{-}=c_{j^{*} i^{*}}^{-}$.

Our proof easily follows from the following elementary, but fundamental, fact.

Lemma. Let $\left\{\beta_{1}, \ldots, \beta_{m}\right\}$ be a basis of a complement to $\operatorname{Hom}_{A}\left(P_{i} / P_{i} J^{n-1}, P_{j}\right)$ in $\operatorname{Hom}_{A}\left(P_{i} / P_{i} J^{n}, P_{j}\right)$. Set

$$
E_{i} / P_{i} J^{n}=\bigcap_{r=1}^{m} \operatorname{ker} \beta_{r}, \quad E_{j}=\sum_{r=1}^{m} \operatorname{Im} \beta_{r} .
$$

Received by the editors August 24, 1982.

1980 Mathematics Subject Classification. Primary 20C05, 16A26; Secondary 16A46.

'This work was supported by the Science \& Engineering Research Council, U. K. and written while the author was visiting the University of Oxford. 
Then

$$
m=\operatorname{dim}\left(\operatorname{Hom}_{A}\left(S_{j}, P_{i} / E_{i}\right)\right)=\operatorname{dim}\left(\operatorname{Hom}_{A}\left(E_{j}, S_{i}\right)\right) .
$$

Proof. The first equality follows from the fact that

$$
m=\operatorname{dim}\left(\operatorname{Hom}_{A}\left(S_{j}, P_{i} J^{n-1} / P_{i} J^{n}\right)\right) .
$$

Moreover, for any $\beta=\Sigma \lambda_{r} \beta_{r}, \beta\left(P_{i}\right)$ is of Loewy length $n$. In particular, if $\pi$ is the canonical homomorphism $E_{j} \rightarrow E_{j} / E_{j} J$, then $\pi \beta_{1}, \ldots, \pi \beta_{m}$ are linearly independent, i.e. $E_{j} / E_{j} J \simeq\left(S_{i}\right)^{m}$, which is equivalent to the second equality.

REMARK. It immediately follows from this observation that the Cartan matrix of $A$ is symmetric. Indeed, if we denote the dimension above by $m_{n}$, we see that $c_{i j}=\Sigma_{n} m_{n}=c_{j i}$, where the first equality follows by considering the Loewy series of $P_{i}$, the second by considering the socle series of $P_{j}$.

THEOREM B. The multiplicity of $S_{j}$ in $P_{i} J^{n-1} / P_{i} J^{n}$ equals that of $S_{i *}$ in $P_{j^{*}} J^{n-1} / P_{j^{*}} J^{n}$ for any $n$.

Proof. Using duality, it suffices to prove that the first number, $m_{1}$, is less than or equal to the second, $m_{2}$. By the Lemma,

$$
m_{1}=\operatorname{dim}\left(\operatorname{Hom}_{A}\left(S_{j}, P_{i} / P_{i} J^{n}\right)\right)=\operatorname{dim}\left(\operatorname{Hom}_{A}\left(B, S_{i}\right)\right),
$$

where $B$ is a submodule of $P_{j}$ of the form $B=\sum_{r=1}^{m} B_{r}$, and each $B_{r}$ is a homomorphic image of $P_{i}$ in $P_{j}$ of Loewy length exactly $n$. But this implies that $B^{*}$ is a quotient module of $P_{j^{*}}$ with $B^{*} J^{n-1}=\left(S_{i^{*}}\right)^{m_{1}}$.

Now, in general, if $0 \rightarrow L \rightarrow M \rightarrow N \rightarrow 0$ is exact, we obtain

$$
L+M J^{n-1} / L+M J^{n} \simeq\left(L+M J^{n-1} / L\right) /\left(L+M J^{n} / L\right) \simeq N J^{n-1} / N J^{n} .
$$

Choosing $M=P_{j^{*}}$ and $N=B^{*}$, we consequently obtain that, indeed, $m_{1} \leqslant m_{2}$.

Proof of Theorem A. We denote the Cartan matrix of $A / J^{n}$ by $\left(c_{i j}(n)\right)$ and use induction on $n$. Thus it suffices to prove that

$$
c_{i j}(n)-c_{i j}(n-1)=c_{j^{*} i^{*}}(n)-c_{j^{*} i^{*}}(n-1),
$$

which is exactly the statement of Theorem $B$.

This result has several applications, of which we just mention one:

COROLlaRY. Let $P_{i_{1}}, \ldots, P_{i_{e}}$ be the projective modules of a self-dual block of $F[G]$. Assume that $P_{i_{1}}$ is self-dual and the Loewy series of $P_{i_{2}}, \ldots, P_{i_{e}}$ are known. Then the Loewy series of $P_{i_{1}}$ is known as well, except for the composition factors isomorphic to $S_{i_{1}}$.

\section{REFERENCES}

1. C. W. Curtis and I. Reiner, Representation theory of finite groups and associative algebras, Wiley, New York, 1962. MR 26 \#2519.

Mathematics Institute, Aarhus University, Aarhus, Denmark

MATHEMATICAL INSTITUTE, OXFoRd, ENGLAND

Current address. School of Mathematics, The Institute for Advanced Study, Princeton, New Jersey 08540 